\title{
Embolic stroke of undetermined source (ESUS) cohort of Brazilian patients in a university hospital
}

\author{
Coorte brasileira de pacientes com AVC embólico de fonte indeterminada (ESUS) em um \\ hospital universitário
}

\begin{abstract}
Gabriel Pinheiro MODOLO', Juli Thomaz De SOUZA', Fernanda Cristina WINCKLER ${ }^{1}$, Natalia Cristina FERREIRA'2, Marcos Cristiano LANGE 3 , Wagner Mauad AVELAR ${ }^{4}$, Marcos Ferreira MINICUCCI ${ }^{5}$, Carlos Clayton Macedo de FREITAS', Paula Schmidt AZEVEDO ${ }^{5}$, Gustavo José LUVIZUTTO' ${ }^{6}$, Leonardo Antônio Mamede ZORNOFF", Silméia Garcia Zanati BAZAN5, Rodrigo BAZAN
\end{abstract}

\begin{abstract}
Embolic stroke of undetermined source (ESUS) is an important group of cryptogenic strokes that are in evidence due recent ongoing trials. We reviewed medical records at discharge from the stroke unit of all patients who met ESUS criteria and attended our institution between February 2016 and July 2017. Among 550 stroke patients, 51 had ESUS. We found that hypertension (60\%), diabetes mellitus (34\%), and smoking (36\%) were the most prevalent risk factors. The mean National Institutes of Health Stroke Scale (NIHSS) scores were 7 at admission and 4 at discharge, while median scores on the modified Rankin scale were 0 and 2 at admission and discharge, respectively. Our sample had similar ages, risk factors prevalence and NIHSS scores at admission and discharge when compared with European and North American cohorts. Although a small cohort, our study suggests that the ESUS population is similar in countries with different health financing.
\end{abstract}

Keywords: Stroke; embolism, embolic stroke of undetermined source.

\section{RESUMO}

Acidentes vasculares cerebrais (AVC) embólicos de fonte indeterminada (ESUS) é um grupo importante de pacientes com AVC criptogênico que estão em evidência devido a recentes ensaios clínicos. Foram revisados os prontuários médicos na alta da unidade de AVC de todos os pacientes que preencheram os critérios para ESUS atendidos em nossa instituição entre fevereiro de 2016 e julho de 2017 . Entre 550 AVCs, 51 eram pacientes com ESUS. Hipertensão (60\%), diabetes mellitus (34\%) e tabagismo (36\%) foram os fatores de risco mais prevalentes. Os escores médios do National Institutes of Health Stroke Scale (NIHSS) foram 7 na admissão e 4 na alta, enquanto os escores médios na escala de Rankin modificada ( $\mathrm{mRs}$ ) foram 0 e 2 na admissão e alta, respectivamente. Nossa amostra teve idade, prevalência de fatores de risco, escores NIHSS na admissão e alta, quando comparados com coortes europeias e norte-americanas semelhantes. Apesar de ser uma pequena coorte, nosso estudo sugere que a população ESUS é semelhante em países com diferentes níveis de financiamento em saúde.

Palavras-chave: Acidente vascular cerebral; embolia, acidentes vasculares cerebrais embólicos de fonte indeterminada.

\footnotetext{
${ }^{1}$ Neurology, Psychiatry and Psycology Department, Botucatu Medical School, São Paulo State University (Unesp), Botucatu, São Paulo, Brazil;

2 Botucatu Medical School, São Paulo State University (Unesp), Botucatu, São Paulo, Brazil;

${ }^{3}$ Neurology Division, Internal Medicine Department, Hospital de Clínicas, Federal University of Paraná, Curitiba PR, Brazil;

${ }^{4}$ Department of Neurology, University of Campinas, Campinas, SP, Brazil;

${ }^{5}$ Internal Medicine Department, Medical School, São Paulo State University (Unesp), Botucatu, São Paulo, Brazil;

${ }^{6}$ Department of Applied Physical Therapy, Federal University of Triângulo Mineiro (UFTM), Uberaba, Brazil.
}

Gabriel Pinheiro Modolo (iD https://orcid.org/0000-0003-1057-5089; Juli Thomaz de Souza iD https://orcid.org/0000-0003-2227-7505; Fernanda Cristina Winckler (iD) https://orcid.org/0000-0002-7299-5612; Natalia Cristina Ferreira iD https://orcid.org/0000-0002-2323-6310; Marcos Cristiano Lange (iD https://orcid.org/0000-0002-0405-7157; Wagner Mauad Avelar (iD https://orcid.org/0000-0002-0025-4225; Marcos Ferreira Minicucci (iD https://orcid.org/0000-0002-5980-4367; Carlos Clayton Macedo de Freitas (iD https://orcid.org/0000-0001-5210-4336; Paula Schmidt Azevedo (iD https://orcid.org/0000-0002-5843-6232; Gustavo José Luvizutto (iD) https://orcid.org/0000-0002-6914-7225; Leonardo Antônio Mamede Zornoff (iD https://orcid.org/0000-0002-9831-8820; Silméia Garcia Zanati Bazan (iD) https://orcid.org/0000-0002-0607-8189; Rodrigo Bazan (iD) https://orcid.org/0000-0003-3872-308X

Correspondence: Gabriel Pinheiro Modolo; Endereço completo; Av. Prof. Mário Rubens Guimarães Montenegro, s/n - UNESP - Campus de Botucatu Botucatu/SP - CEP 18618687; E-mail: g.modolo@unesp.br

Conflict of interest: There is no conflict of interest to declare.

Received 11 May 2018; Received in final form 22 November 2018; Accepted 20 January 2019. 
Cryptogenic strokes are thought to comprise $25-40 \%$ of all ischemic strokes, accounting for approximately 400,000 cases each year in western Europ ${ }^{1,2}$. Moreover, in patients under 55 years of age, almost half of all strokes are cryptogenic ${ }^{3}$. Although recurrence rates for cryptogenic stroke are close to those of large artery and cardioembolic causes ${ }^{4,5}$ cryptogenic stroke populations tend to be highly variable. This variation makes comparative analyses difficult and may result in nonspecific or conflicting data regarding secondary stroke prevention and long-term outcomes ${ }^{3}$. One major reason for this is that strokes with more than one possible cause and strokes with no identifiable cause are grouped together. However, even after adjusting for higher rates of treatment with intravenous tissue plasminogen activator, Scullen et al. ${ }^{4}$ reported that patients with ischemic stroke, for which no cause could be identified, experienced better outcomes than other Trial of Org 10172 in Acute Stroke Treatment (TOAST) classification groups, such as embolic or large vessel strokes ${ }^{4,5}$.

\section{Definition}

Based on recent evidence, which suggests that most cryptogenic strokes are thromboembolic, Hart et al. proposed the concept of "embolic stroke of undetermined source (ESUS)" along with the basic methods for the etiologic investigation of this diagnosis ${ }^{1}$. This report proved critical due to the large number of patients whose conditions aligned with this concept; a recent review of nine studies reported that the prevalence of ESUS ranges from 9-25\%, averaging 17\% (i.e., approximately one in six) of all ischemic strokes ${ }^{6}$. The investigation was carried out by obtaining images of the cerebral parenchyma and the intracranial/extracranial vessels, obtaining a transthoracic echocardiogram, obtaining an electrocardiogram, and performing 24-hour ambulatory Holter monitoring or 24-hour cardiac telemetry. During the investigation, diagnoses such as vasculitis, endocarditis, lupus erythematosus, arterial dissection, and neoplasms were ruled out. To exclude small vessel disease, the image of the parenchyma also had to be larger than $1.5 \mathrm{~cm}^{1}$.

There is still a debate regarding whether other, less common causes of stroke should be ruled out. To date, no systematic investigation of carotid arch or interatrial communications has been proposed in the protocols for ESUS investigations or performed in recent trials concerning the treatment of these strokes ${ }^{6,7,8,9}$. However, the concept of ESUS is thought to expedite decisions regarding treatment, aid in the prevention of secondary strokes, and reduce the cost and number of examinations.

Embolic stroke of undetermined source refers to nonlacunar, cryptogenic ischemic strokes for which embolism is the most likely mechanism ${ }^{1,6}$. Because some researchers have hypothesized that anticoagulants may be more effective than antiplatelet agents for secondary stroke prevention in patients with ESUS, interest in these medications has been fueled by two ongoing randomized controlled trials and one recently-published trial comparing nonvitamin $\mathrm{K}$ antagonists/direct-acting oral anticoagulants versus aspirin for secondary stroke prevention ${ }^{6,7,8}$. Our center participated in two of these trials. We believe that the findings of these studies are of great interest, as there are few studies on the prevalence, risk factors, and functional outcomes in Latin American patients with ESUS. The present study included a cohort of such patients.

As a middle-income country, there are few centers that are properly structured to receive stroke patients in Brazil. According to a recent public consultation with neurologists and neurosurgeons by the Brazilian Federal Medical Council, only $22 \%$ of the public hospitals had adequate conditions to attend to stroke patients, and only $3 \%$ were considered completely adequate ${ }^{10}$. In our institution, we have a comprehensive stroke unit. Patients admitted to our unit receive all the necessary care for the hyperacute phase of stroke; rehabilitation and an etiological investigation are also started during the unit stay. The majority of patients have their etiology and secondary prevention already defined at discharge. This is not the reality in most centers and, therefore, we think the data presented here are very representative, as there is minimal loss of patients, due to the comprehensive inpatient investigation.

\section{METHODS}

\section{Patients and data collection}

We retrospectively reviewed the medical records and discharge summaries of all patients treated in our stroke unit who met the diagnostic criteria for ESUS, as defined by the Cryptogenic Stroke/ESUS International Working Group ${ }^{1}$, and who stayed at the Hospital of Clinics Botucatu Medical School between February 2016 and July 2017. The present study was approved by the local ethics committee on human research. No patients were participating in clinical trials at the time that the data were collected. Patients were diagnosed with ESUS following the criteria and investigation protocol proposed by Hart et al., as previously described ${ }^{1}$.

\section{Investigation protocol}

The investigation protocol at our institution was based on the TOAST classification. All patients underwent a cerebral computed tomography scan upon admission, while some underwent an additional scan after 24 hours. Depending on the clinical evaluation, an MRI was performed for patients with strokes from the posterior circulation or with a dubious diagnosis. If the patient arrived within the first eight hours after the ictus, angiotomography of the cerebral and cervical arteries was performed. If the patient arrived more than eight hours after the ictus, a duplex scan of the cervical arteries and a transcranial Doppler were performed. When the clinical evaluation called for it, the study was completed with an 
anatomic examination and angiotomography or digital angiography. A transthoracic echocardiogram was performed on all patients. A transesophageal echocardiogram was ordered when a right-to-left shunt was suspected or observed during the transthoracic exam or when other findings needed additional clarification. All patients underwent electrocardiography upon admission and had 24 hours of cardiac monitoring. Holter monitoring was performed for 24 hours when the patients were above 55 years of age, when arrhythmias were suspected, and when the stroke origin had been classified as cryptogenic. Laboratory tests were also performed for syphilis, Chagas disease, glycated hemoglobin, thyroid stimulating hormone, total cholesterol, fractionated cholesterol, and triglycerides. An autoimmune panel was also performed for patients under 55 years of age, and for prothrombotic states, anticardiolipin, lupus anticoagulant, factor V Leiden, proteins $\mathrm{S}$ and $\mathrm{C}$, and antithrombin III were requested.

\section{Statistical analysis}

Data are presented as the median and standard deviation or median and 25th/75th percentiles. Categorical variables were analyzed using chi-squared or Fisher's exact test, while parametric data were analyzed using Student's t-test. The Mann-Whitney U-test was used for nonparametric data. The level of statistical significance (p) was set at $5 \%$.

\section{RESULTS}

Approximately 550 patients were treated during the 18 months of the study. Hemorrhagic strokes accounted for $10 \%$ of patients treated, while cerebral venous thrombosis accounted for $2 \%$ of the patients. All other strokes were classified as ischemic. Among the 63 patients initially defined with ESUS, five were excluded due to the absence of Holter or telemetry data, while six were excluded following posterior identification of atrial fibrillation or a cause of stroke meeting criteria for another TOAST subgroup (Figure). Also, 15\% of our patients had not undergone the complete investigation protocol and were classified as cryptogenic strokes in an undetermined TOAST subgroup and excluded from the final analyses.

Among the 550 patients treated at our stroke center during the study period, 51 were diagnosed with ESUS (56\% women, mean age: 63 years, ranging from 33-89 years), in accordance with the classification criteria proposed by Hart et al. ${ }^{1}$. Among these patients, hypertension (60\%), diabetes mellitus (34\%), and smoking (36\%) were the most prevalent risk factors. The mean National Institutes of Health Stroke Scale (NIHSS) scores were 7 at admission, and 4 at discharge, while the median scores on the modified Rankin scale (mRs) were 0 prestroke and 2 at discharge. Approximately $19.5 \%$ of patients went to sleep unaffected and awoke with stroke symptoms (i.e., wake-up stroke). In addition, stroke accounted for the first cerebrovascular event in $83 \%$ of patients. In the clinic, $39 \%$ of patients presented with lacunar syndromes, while $93 \%$ presented with syndromes of the anterior circulation. Most patients were not receiving treatment for ischemic events with prophylactic medication (76\%), and only $17 \%$ were on acetylsalicylic acid (ASA) treatment before the stroke. All patients received treatment with antiaggregants after discharge,

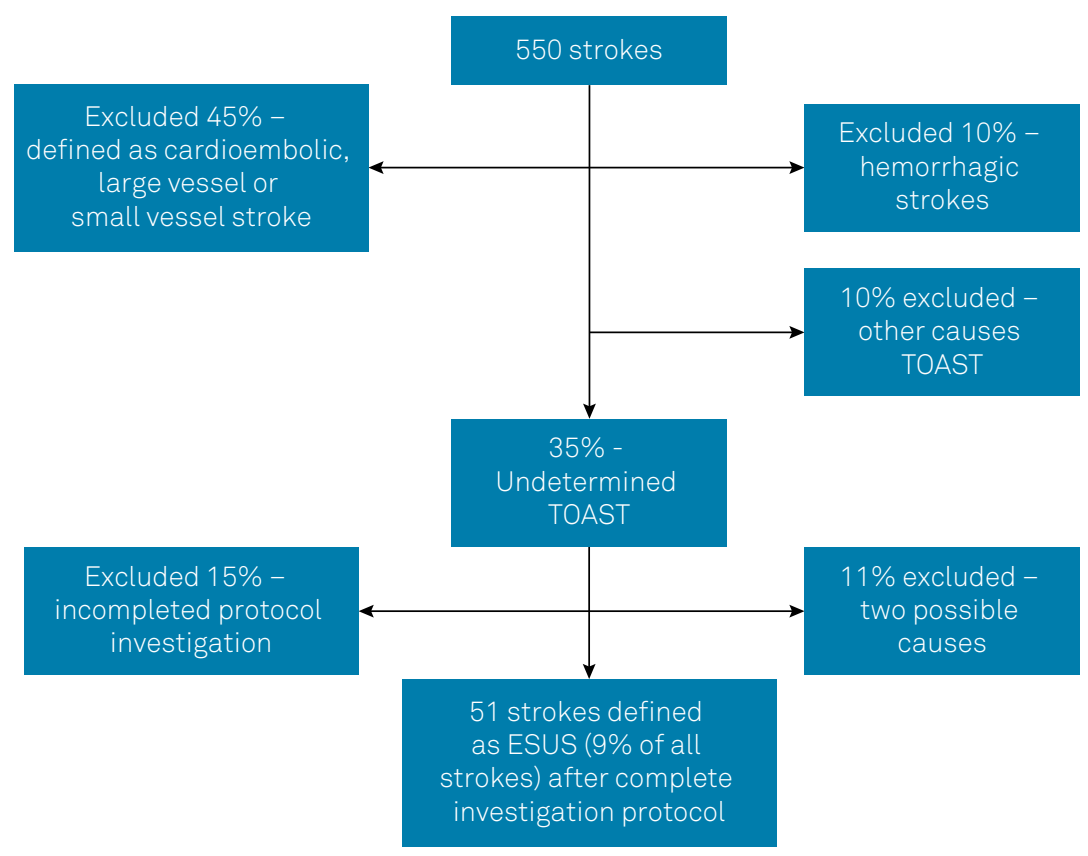

Figure. Frequency of embolic strokes of undetermined source (ESUS). 
some of whom changed to anticoagulant treatment in the outpatient setting (67.4\% using ASA and $28.3 \%$ using ASA + clopidogrel). Only five patients presented with an intracardiac shunt as confirmed via transesophageal echocardiography or transcranial Doppler (but with low conductance). As shown in Table 1, when these data were analyzed, only NIHSS prestroke scores were significantly associated with better outcomes (i.e., mRs of $0-2$ at discharge) $(p<0.05)$. Demographic variables and their relationship with $\mathrm{mRs}$ are summarized in Table 2.

Table 1. Demographic data of the overall sample.

\begin{tabular}{|c|c|c|}
\hline Variable & $\mathrm{n}$ & $\%$ \\
\hline Age (years) & $62.9(31-89)$ & - \\
\hline Sex (male) & 22 & 43.13 \\
\hline NIHSS score prestroke (median) & $7.5(0-25)$ & - \\
\hline NIHSS score at discharge (median) & $3.7(0-16)$ & - \\
\hline Prior stroke & 11 & 21.56 \\
\hline Wake-up stroke & 12 & 23.52 \\
\hline Hypertension & 33 & 64.70 \\
\hline Diabetes & 19 & 37.25 \\
\hline Smoking & 19 & 37.25 \\
\hline Drinking & 11 & 21.56 \\
\hline mRs at admission (0 - 2) & 48 & 94.11 \\
\hline mRs at discharge $(0-2)$ & 18 & 35.29 \\
\hline Prior use of antiplatelet & 13 & 25.49 \\
\hline Thrombolyzed patients & 9 & 17.64 \\
\hline
\end{tabular}

mRs: modified Rankin scale; NIHSS: National Institute of Health Stroke Scale.

\section{DISCUSSION}

\section{Risk factors}

Cryptogenic strokes account for approximately $25 \%$ of stroke cases. Among these patients, those with ESUS can be identified using an objective investigation protocol. Although ESUS may be associated with various causes-such as aortic arch atheroma and patent foramen ovale (PFO)-investigation protocols differ among services, and there are still many concerns regarding the treatment and prevention of new strokes in patients with less common diseases.

It is still questionable whether it is better for those patients to insist on more intensive investigation protocols or to be more aggressive in empiric secondary prophylaxis with anticoagulants instead of antiaggregates, assuming a probable embolic origin. The fact that almost $10 \%$ of our initial cohort was excluded because of a diagnosis of atrial fibrillation reinforces this theory. The first option is reinforced by studies such as the one by Sanna et al. ${ }^{11}$ that showed a greater incidence of atrial fibrillation using an implantable cardiac monitoring device compared with a traditional cardiac rhythm investigation. In addition, screening using the transcranial Doppler bubble study is a reasonable, sensitive and noninvasive test for an investigation of a PFO in young patients before transesophageal echocardiography. Although some authors have argued that more aggressive prophylactic treatment is more reasonable than the risk of recurrence, this opinion is being questioned after the publication of negative results by the Navigate-ESUS study that showed a lack of a benefit of rivaroxaban compared with ASA and a higher risk of hemorrhage ${ }^{7}$. These negative results and the fact that the ESUS definition is an umbrella term for patients with very

Table 2. Clinical and demographic variables in patients with ESUS and relation with mRs at discharge.

\begin{tabular}{|c|c|c|c|}
\hline \multirow{2}{*}{ Variables } & \multicolumn{2}{|c|}{$\mathrm{mRs}$ at discharge } & \multirow{2}{*}{$\mathrm{p}$-value } \\
\hline & $0-2(n=33)$ & $3-5(n=18)$ & \\
\hline Age, (years) & $62.5 \pm 13.0$ & $63.8 \pm 13.1$ & 0.719 \\
\hline Age $>55$ years, $n^{\circ}(\%)$ & $9(27.3)$ & $4(22.2)$ & 0.750 \\
\hline Female sex, $n^{\circ}(\%)$ & $19(57.6)$ & $10(55.6)$ & 0.876 \\
\hline Time from ictus $>6$ hours, $n^{\circ}(\%)$ & $15(45.5)$ & $9(50.0)$ & 0.839 \\
\hline Time from ictus $>24$ hours, $n^{\circ}(\%)$ & $5(15.2)$ & $4(22.2)$ & 0.466 \\
\hline NIHSS prestroke & $4.0(2.0-8.5)$ & $10.5(10.8-15.3)$ & 0.011 \\
\hline NIHSS at discharge & $2.0(0.5-2.0)$ & $6.5(3.8-11.3)$ & $<0.001$ \\
\hline Thrombolysis, $n^{\circ}(\%)$ & $5(15.2)$ & $4(22.2)$ & 0.703 \\
\hline 1st event, $n^{\circ}(\%)$ & $27(81.8)$ & $14(77.8)$ & 0.727 \\
\hline \multicolumn{4}{|l|}{ mRs at admission, $n^{\circ}(\%)$} \\
\hline 0 & $27(81.8)$ & $12(66.7)$ & \multirow{4}{*}{0.045} \\
\hline 1 & $6(18.2)$ & $2(11.1)$ & \\
\hline 2 & $0(0)$ & $1(5.6)$ & \\
\hline 3 & $0(0)$ & $3(16.6)$ & \\
\hline
\end{tabular}


different etiologic profiles, including cardiac sources such as paroxysmal atrial fibrillation and atherothrombotic sources such as aortic plaques, that are very different in prognostic value and response to treatment, have been responsible for the questioning of the use of the classification as ESUS.

Our patients were of similar median age to those examined in previous studies. Hart et al. reported a median age of 65 years in a review of nine studies conducted in various countries, including Mexico ${ }^{6}$. Perera et al. reported a mean age of 62 years in the ESUS global registry ${ }^{12}$. Although Arauz et al. ${ }^{5}$ found a median age of 44 years in a Mexican cohort of consecutive patients with ESUS, the median age for all patients with cryptogenic stroke was 66 years, and some authors have argued that the median age of patients with ESUS may be lower than that of patients with other forms of cryptogenic stroke. Brazil is a country known for a high incidence of stroke in young patients and a portion of these patients are presenting with an increasingly high incidence of hypertension, diabetes and obesity. For this reason, we thought our results would show a lower median age for our patients. Perhaps the reason is that a profile of another TOAST category, such as small or large vessel disease, includes young patients who already have complications related to hypertension, diabetes, alcoholism and smoking.

Although the percentage of men in our cohort was higher than that in the global registry, this percentage was similar to that observed in the Latin American population $(58 \%)^{12}$. In addition, the nine studies reviewed by Hart et al. ${ }^{6}$ included a greater number of men, with the exception of the study by Masina et al. as referenced by Hart et al. ${ }^{6}$.

Our analyses revealed a similar prevalence of risk factors such as hypertension in our registry and worldwide (60\%), as determined by Perera et al. ${ }^{12}$. However, rates of diabetes and smoking were higher in our cohort compared with Perera et al. ${ }^{12}$. Interestingly, the differences increased when only the Latin American population was compared ${ }^{12}$, and all risk factors were less prevalent in the Arauz et al. cohort reported prevalence rates ranging from $25 \%$ to $74 \%$ for hypertension and $7 \%$ to $32 \%$ for diabetes ${ }^{5,6,12}$.

\section{Presentation and therapy}

In our study, the median NIHSS score at admission was the same as that observed in the Mexican cohort examined by Arauz et al. ${ }^{5}$, yet higher than that observed less than 48 hours after ictus in the global registry (NIHSS $=4)$. Lower median NIHSS scores were also observed for all studies reviewed by Hart et al. ${ }^{6}$. In our cohort, the median NIHSS score at discharge and mRs prior to the event and at discharge were similar to those observed for patients in the ESUS global registry $^{12}$. Arauz et al. reported that $59.4 \%$ of patients exhibited mRs scores of 2 or less and that ESUS was associated with better outcomes than other types of cryptogenic strokes ${ }^{5}$. The relatively low NIHSS scores at admission that were found in ESUS patients, and the better functional outcomes reported by some authors when compared with cardioembolic strokes ${ }^{4,5}$, are very interesting facts. In a group thought to be associated with embolic mechanisms, one might have assumed higher NIHSS scores and outcomes similar to those of cardioembolic origin associated with large core and poor collateral infarcts. These data reinforce the view that ESUS may be a group too heterogeneous to simplify for one medication or protocol.

Antiplatelet therapy was continued at discharge in all patients in the present study. Some patients with NIHSS scores $<5$ or a transient ischemic attack with an ABCD2 score $\geq 4$ underwent dual antiplatelet therapy (ASA + clopidogrel), while others were included in subsequent randomized trials of anticoagulation versus antiaggregation. In all studies, the majority of patients with ESUS were treated via antiplatelet therapy at the time of discharge $e^{5,6,12}$. Only $17 \%$ of patients were on ASA treatment before hospital admission, while none had received treatment with anticoagulants prior to the index stroke in our cohort, which is less than the $31 \%$ on antiplatelet treatment and $1 \%$ on anticoagulants reported by Perera et al. ${ }^{12}$. These findings cast doubt on the most appropriate treatment for patients with ESUS; although the majority leave the hospital on antiplatelet agents, substantial evidence suggests that these patients may benefit from anticoagulation treatment. Almost one-third of patients in the global registry experienced a stroke during treatment with antiplatelet agents, while almost one-fifth in our cohort had experienced a prior stroke (17\%) while using antiplatelets. The Navigate-ESUS results highlighted the importance of this matter. We hope that the ongoing trials will shed further light on this issue ${ }^{7,8,9}$.

Almost one in five patients experienced wake-up strokes in our cohort; these findings are interesting because wakeup strokes are classically thought to be caused by thrombosis in situ and not by embolism due to early diurnal peaks of prothrombotic hematological parameters, such as plasminogen activator inhibitor-1 and platelet aggregation function ${ }^{13,14,15}$. Some authors, such as Riccio et al. ${ }^{16}$, found a three-fold increase in the odds of a new diagnosis of atrial fibrillation in patients presenting with wake-up strokes and transient ischemic attacks. Wake-up strokes are thought to be related to changes in homeostasis caused by circadian cycles, and these may contribute to different mechanisms of strokes ${ }^{16}$.

Although we identified only five patients with intracardiac communication, this number may have been higher, as we did not systematically test for shunt detection. Perera et al. reported a prevalence as high as $25 \%$ for PFO worldwide and as high as $42 \%$ for their Latin American cohort ${ }^{12}$. They also reported that the prevalence of aortic arch plaque, when evaluated via transesophageal echocardiography, was 29\%. Using contrast-enhanced cardiac magnetic resonance imaging, Takasugi et al. ${ }^{17}$ identified thrombi in the left ventricles of 12 out of 60 patients with ESUS. However, when transthoracic 
echocardiography was used, thrombi were identified in only one of these patients ${ }^{17}$. These data reinforce the embolic pathophysiology, as more extensive investigations have found sources of thrombi in more patients.

The only variable associated with improved functional outcomes was a lower NIHSS score before stroke. Although thrombolysis was not associated with improvements in functional outcomes, this may have been due to our small sample size and transversal evaluation.

Patients with ESUS represent an important population whose therapeutic management is currently being investigated in several clinical trials. However, few researchers have attempted to investigate the epidemiology of these strokes in Latin American cohorts. Our findings further underscore the need to investigate cases of ESUS in young Brazilian patients with cryptogenic stroke. Although a small cohort, we found similar results when compared with ESUS populations in previous studies from different countries, although whether the patients would be better assisted by a more aggressive investigation protocol or more aggressive treatment remains in question. The recent results of the Navigate trial, which showed a greater incidence of major bleeding and no difference in stroke prevention with oral anticoagulation, reinforce the need for better characterization of different stroke mechanisms in ESUS and more caution in aggressive treatment regimens. We also think that protocols that involve methods such as transcranial Doppler and transesophageal echocardiography may be a good and inexpensive option for a better understanding and individualization of treatment and prevention in the face of crises.

Our results were similar of those in previous reports of multicentric studies, however the underlying mechanisms of ESUS remains obscure and a more aggressive investigation in the acute phase is necessary.

\section{References}

1. Hart RG, Diener HC, Coutts SB, Easton JD, Granger CB, O'Donnell $\mathrm{MJ}$, et al. Embolic strokes of undetermined source: The case for a new clinical construct. Lancet Neurol [Internet]. 2014;13(4):429-38. Available from: $\mathrm{http}: / / \mathrm{dx}$.doi.org/10.1016/S1474-4422(13)70310-7

2. Amarenco P. Cryptogenic Stroke, Aortic Arch Atheroma, Patent Foramen Ovale, and the Risk of Stroke. Cerebrovasc Dis. 2005;20(2):68-74.

3. Li L, Yiin GS, Geraghty OC, Schulz UG, Kuker W, Mehta Z, et al. Incidence, outcome, risk factors, and long-term prognosis of cryptogenic transient ischaemic attack and ischaemic stroke: A population-based study. Lancet Neurol [Internet]. 2015;14(9):903-13. Available from: http://dx.doi.org/10.1016/\$1474-4422(15)00132-5

4. Scullen TA, Monlezun DJ, Siegler JE, George AJ, Schwickrath M, El Khoury R, et al. Cryptogenic stroke: Clinical consideration of a heterogeneous ischemic subtype. J Stroke Cerebrovasc Dis [Internet]. 2015;24(5):993-9. Available from: http://dx.doi. org/10.1016/j.jstrokecerebrovasdis.2014.12.024

5. Arauz A, Morelos E, Colín J, Roldán J, Barboza MA. Comparison of functional outcome and stroke recurrence in patients with embolic stroke of undetermined source (ESUS) vs. Cardioembolic stroke patients. PLoS One. 2016;11(11):1-9.

6. Hart RG, Catanese L, Perera KS, Ntaios G, Connolly SJ. Embolic Stroke of Undetermined Source: A Systematic Review and Clinical Update. Stroke. 2017;48(4):867-72.

7. Hart RG, Sharma M, Mundl H, Kasner SE, Bangdiwala SI, Berkowitz SD, et al. Rivaroxaban for Stroke Prevention after Embolic Stroke of Undetermined Source. N Engl J Med [Internet]. 2018;378(23):2191-201. Available from: http://www.nejm.org/ doi/10.1056/NEJMoa1802686

8. Diener HC, Donald Easton J, Granger CB, Cronin L, Duffy C, Cotton D, et al. Design of Randomized, double-blind, Evaluation in secondary Stroke Prevention comparing the EfficaCy and safety of the oral Thrombin inhibitor dabigatran etexilate vs. acetylsalicylic acid in patients with Embolic Stroke of Undetermined Source (RE-SPECT ESUS). Int J Stroke. 2015;10(8):1309-12.
9. Geisler T, Poli S, Meisner C, Schreieck J, Zuern CS, Nägele T, et al. Apixaban for treatment of embolic stroke of undetermined source (ATTICUS randomized trial): Rationale and study design. Int J Stroke. 2017;12(9):985-90.

10. Conselho Federal de Medicina - CFM. . Infraestrutura para assistência 287 ao AVC no SUS é inadequada, apontam 76\% dos especialistas. [Internet]. [cited 2017 Jul 17]. Available from: https://portal.cfm.org.br/index.php?option=com_content\&view=arti cle\&id =27054:2017-07-17-17-38-24\&catid =3

11. Sanna T, Diener H-C, Passman RS, Di Lazzaro V, Bernstein RA, Morillo CA, et al. Cryptogenic Stroke and Underlying Atrial Fibrillation. N Engl J Med. 2014;370(26):2478-86.

12. Perera KS, Vanassche T, Bosch J, Giruparajah M, Swaminathan B, Mattina KR, et al. Embolic strokes of undetermined source: Prevalence and patient features in the ESUS Global Registry. Int J Stroke. 2016;11(5):526-33.

13. Scheer FAJL, Shea SA. Human circadian system causes a morning peak in prothrombotic plasminogen activator inhibitor-1 (PAI-1) independent of the sleep/wake cycle. Blood. 2014;123(4):590-3.

14. Bonten TN, Snoep JD, Assendelft WJ, Zwaginga JJ, Eikenboom J, Huisman MV, Rosendaal FR van der BJ. Time-Dependent Effects of Aspirin on Blood Pressure and Morning Platelet Reactivity A Randomized Cross-Over Trial. Hypertension. 2015;65:743-50.

15. Scheer FAJL, Michelson AD, Frelinger AL, Evoniuk H, Kelly EE, McCarthy M, et al. The human endogenous circadian system causes greatest platelet activation during the biological morning independent of behaviors. PLoS One. 2011;6(9).

16. Riccio PM, Klein FR, Cassara FP, Giacomelli FM, Gonzalez Toledo ME, Racosta JM, et al. Newly diagnosed atrial fibrillation linked to wake-up stroke and TIA: Hypothetical implications. Neurology. 2013;80(20):1834-40.

17. Takasugi J, Yamagami H, Noguchi T, Morita Y, Tanaka T, Okuno Y, et al. Detection of Left Ventricular Thrombus by Cardiac Magnetic Resonance in Embolic Stroke of Undetermined Source. Stroke. 2017;48(9):2434-40. 